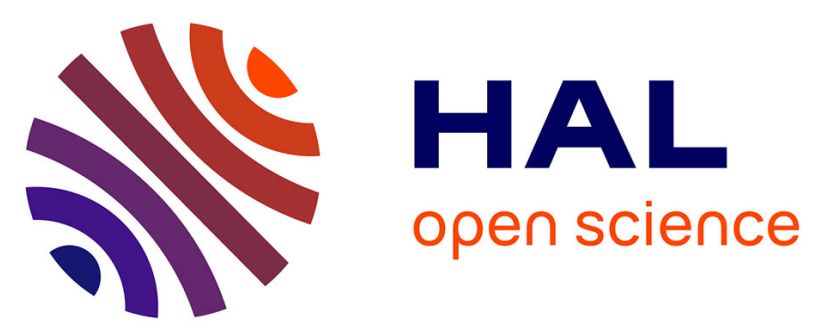

\title{
APPLICATION OF THE WAVE FINITE ELEMENTS FOR CALCULATING DYNAMIC RESPONSES OF 2D STRUCTURES OF ARBITRARY SHAPES SUBJECTED TO EXTERNAL LOADS
}

Tien Hoang, Denis Duhamel, Gilles Forêt

\section{- To cite this version:}

Tien Hoang, Denis Duhamel, Gilles Forêt. APPLICATION OF THE WAVE FINITE ELEMENTS FOR CALCULATING DYNAMIC RESPONSES OF 2D STRUCTURES OF ARBITRARY SHAPES SUBJECTED TO EXTERNAL LOADS. Compdyn 2019, Jun 2019, Crète, Greece. hal-02915352

HAL Id: hal-02915352

https://hal.science/hal-02915352

Submitted on 14 Aug 2020

HAL is a multi-disciplinary open access archive for the deposit and dissemination of scientific research documents, whether they are published or not. The documents may come from teaching and research institutions in France or abroad, or from public or private research centers.
L'archive ouverte pluridisciplinaire HAL, est destinée au dépôt et à la diffusion de documents scientifiques de niveau recherche, publiés ou non, émanant des établissements d'enseignement et de recherche français ou étrangers, des laboratoires publics ou privés. 


\title{
APPLICATION OF THE WAVE FINITE ELEMENTS FOR CALCULATING DYNAMIC RESPONSES OF 2D STRUCTURES OF ARBITRARY SHAPES SUBJECTED TO EXTERNAL LOADS
}

\author{
Tien Hoang ${ }^{1}$, Denis Duhamel ${ }^{1}$, and Gilles Foret ${ }^{1}$ \\ ${ }^{1}$ Laboratoire Navier \\ UMR 8205, Ecole des Ponts ParisTech, IFSTTAR, CNRS, UPE \\ 6-8 av. Blaise Pascal, Cite Descartes, 77420 Champs-sur-Marne, France \\ e-mail: \{tien.hoang, denis.duhamel, gilles.foret\}@enpc.fr
}

Keywords: Wave Finite Element, Dynamic, Vibration, Periodic structure, Waveguide

\begin{abstract}
The wave finite element method (WFE) has been developed originally for one dimensional periodic structures with advantage in calculation time. However, this method cannot apply easily for $2 D$ structures of arbitrary shape. This communication presents a new technique of WFE to calculate the dynamic responses of such a structure subjected to external loads. The structure is decomposed into rectangular domains which can be considered as periodic structures subjected to external loads and nodal reaction forces at the domains boundaries. Then, by using the WFE for theses domains, we can obtain a relation between the external loads, the DOF and the nodal reaction forces at the boundaries of the domains. Finally, by combining this relation with the dynamic equation of the rest of the structure, we obtain an equation of the whole structure to compute its response. This technique permits to reduce all the DOF of the rectangular domains of the structure. Examples showing the efficiency of the method are presented.
\end{abstract}




\section{INTRODUCTION}

The wave finite element method has been developped originally for the wave propagation along one-dimensional periodic elastic structures [1]. From the finite element method for a period of the structure, we can obtain a relation (a transform) between the left and right boundaries of the period. This relation leads to a wave base which is obtain from the eignenvectors of the transform. Then, the response at the boundary of a period can be decomposed in this base to compute with different approaches [2]. Recently, this method has been applied for many different problem of periodic structures [3, 4, 5, 6, 7, 8, 9, 10]. For 2D structures, WFE has been developed by using superelements which are rectangular subdomains[11]. By considering each rectangular domain as a periodic structure, this technique permits to obtain the wave decompositions of the domain responses and to combine in the global dynamic equation. However, this technique cannot be applied easily when the structure is subjected to complex external loads or density loads.

In this article, we present another technique of WFE using superelements for $2 \mathrm{D}$ structures subjected to external loads. Let's consider a 2D structure containing a rectangular domain $\mathcal{P}$ with rectangular elements as shown in Figure 1 and the rest domain is denoted by $\mathcal{R}$. The domain $\mathcal{P}$ is subjected to external density force $\mathbf{F}_{E}$ and the reaction force $\mathbf{F}_{\partial}$ of the domain $\mathcal{R}$ at the common boundary of the two domains. We will use the wave finite element method to obtain a relation between the forces $\mathbf{F}_{E}, \mathbf{F}_{\partial}$ and the DOF at the boundary of the two domains $\mathbf{q}_{\partial}$.

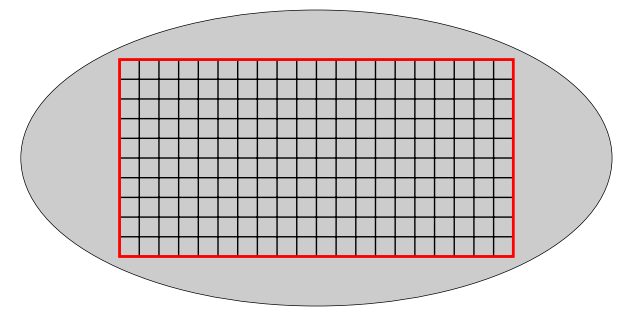

Figure 1: Structure including a rectangular domain $\mathcal{P}$ and the rest $\mathcal{R}$

The rectangular domain $\mathcal{P}$ is a periodic structure of $N$ periods where each one is a column of elements with only nodes on the left and right boundaries as shown in Figure 2. The nodes of $\mathcal{P}$ are denoted by each column $(n)$ with $0 \leq n \leq N$. From the dynamic equation of each

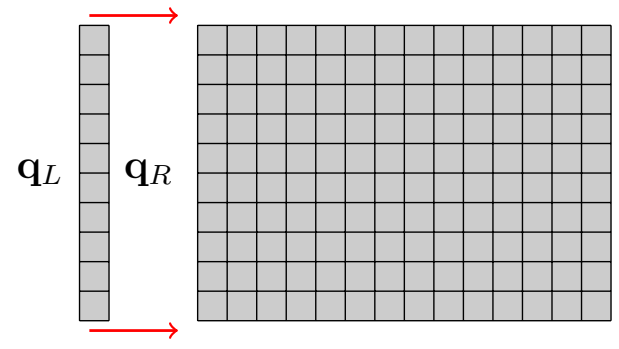

Figure 2: A periodic structure subjected to external loads

column, we get the relation $\mathbf{u}_{R}=\mathbf{S} \mathbf{u}_{L}$ with $\mathbf{u}_{*}$ is the column vector of DOF $\mathbf{q}_{*}$ and nodal loads $\mathbf{F}_{*}$. Moreover, the right boundary of the column $(n)$ is also the left boundary of the column $(n+1)$ and we have

$$
\mathbf{F}_{L}^{(n+1)}+\mathbf{F}_{R}^{(n)}=-\mathbf{F}_{B}^{(n)}
$$


where $\mathbf{F}_{B}^{(n)}$ are the loads applying on the common boundary of $(n)$ and $(n+1)$. Then, by using the wave analysis approach of the wave finite element method [12], we can obtain the wave bases $\Phi, \Phi^{\star}$ with eigenvalues $\boldsymbol{\mu}$ of the transform S. The wave decomposition of the response can be written as follows

$$
\mathbf{q}^{(n)}=\boldsymbol{\Phi}_{q} \boldsymbol{\mu}^{n} \mathbf{Q}-\boldsymbol{\Phi}_{q}^{\star} \boldsymbol{\mu}^{N-n} \mathbf{Q}^{\star}+\boldsymbol{\Phi}_{q} \sum_{k=1}^{n} \boldsymbol{\mu}^{n-k} \mathbf{Q}_{B}^{(k)}+\boldsymbol{\Phi}_{q}^{\star} \sum_{k=n+1}^{N} \boldsymbol{\mu}^{k-n} \mathbf{Q}_{B}^{\star(k)}
$$

where $\mathrm{Q}, \mathrm{Q}^{\star}$ are the wave amplitudes of the left and right ends of the domain and $\mathrm{Q}_{B}, \mathrm{Q}_{B}^{\star}$ are the wave amplitude of the external loads which are calculated by

$$
\begin{aligned}
& \mathbf{Q}_{B}^{(k)}=\boldsymbol{\Phi}_{q}^{\star T} \mathbf{F}_{B}^{(k)} \\
& \mathbf{Q}_{B}^{\star(k)}=\boldsymbol{\Phi}_{q}^{T} \mathbf{F}_{B}^{(k)}
\end{aligned}
$$

where $\mathbf{F}_{B}^{(k)}$ is nodal loads on the right boundary of the period. Thus, we have

$$
\mathbf{q}^{(n)}=\boldsymbol{\Phi}_{q} \boldsymbol{\mu}^{n} \mathbf{Q}-\boldsymbol{\Phi}_{q}^{\star} \boldsymbol{\mu}^{N-n} \mathbf{Q}^{\star}+\boldsymbol{\Phi}_{q} \sum_{k=1}^{n} \boldsymbol{\mu}^{n-k} \boldsymbol{\Phi}_{q}^{\star T} \mathbf{F}_{B}^{(k)}+\boldsymbol{\Phi}_{q}^{\star} \sum_{k=n+1}^{N} \boldsymbol{\mu}^{k-n} \boldsymbol{\Phi}_{q}^{T} \mathbf{F}_{B}^{(k)}
$$

Equation (3) presents the response of the rectangular domain in function of the external loads applying on this domain and the wave ampltudes at the left and right ends of the domain.

\section{FORMULATIONS}

\subsection{Wave analysis of rectangular domains}

Now we will decompose the nodal loads $\mathbf{F}_{B}^{(k)}$ in function of the external loads and the reaction force of the domain $\mathcal{R}$. For $0<n<N$, we can write

$$
\mathbf{F}_{B}^{(n)}=\mathbf{F}_{E}^{(n)}+\tilde{\mathbf{F}}^{(n)}
$$

where $\mathbf{F}_{E}^{(n)}$ are external loads at the column $n$ and $\tilde{\mathbf{F}}^{(n)}$ is the reaction force of the domain $\mathcal{R}$ applying on the periodic domain which is almost zeros except the two nodes at the common boundary of the column $n$ and the domain $\mathcal{R}$.

For the left boundary $\partial L$ of the periodic domain $\mathcal{P}$, we have

$$
\mathbf{F}^{(0)}=-\mathbf{F}_{L}^{(1)}=\mathbf{F}_{E}^{(0)}+\mathbf{F}_{\partial L}
$$

where $\mathbf{F}_{\partial L}$ is the reaction of $\mathcal{R}$ at $\partial L$.

For the right boundary $\partial R$ of the periodic domain $\mathcal{P}$, we need to find out the expression of the reaction force $\mathbf{F}_{\partial R}$ in function of $\mathbf{F}^{(N)}$ which is the nodal load of the left boundary of period $(N+1)$ (see Figure 3). We have

$$
\begin{aligned}
& \mathbf{F}^{(N)}=-\mathbf{F}_{L}^{(N+1)}=-\left(\mathbf{F}_{\partial R}-\dot{\mathbf{F}}^{(N)}\right) \\
& \mathbf{F}_{B}^{(N)}=\mathbf{F}_{E}^{(N)}+\dot{\mathbf{F}}^{(N)}
\end{aligned}
$$

where $\dot{\mathbf{F}}^{(N)}$ is the reaction forces of at the upper and lower node of $\partial R$. By combining equations in (6), we obtain

$$
\mathbf{F}_{B}^{(N)}-\mathbf{F}^{(N)}=\mathbf{F}_{E}^{(N)}+\mathbf{F}_{\partial R}
$$




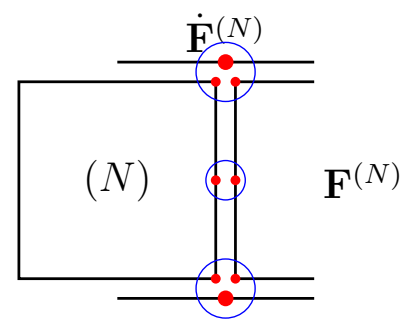

Figure 3: Nodal loads at the column $(N)$ (right boundary of $\mathcal{P}$ )

In addition, we obtain the following results from the definition of the wave amplitude [12]

$$
\begin{aligned}
\mathbf{Q} & =\boldsymbol{\Phi}^{\star T} \mathbf{J} \mathbf{u}^{(0)}=\boldsymbol{\Phi}_{q}^{\star T} \mathbf{F}^{(0)}-\boldsymbol{\Phi}_{F}^{\star T} \mathbf{q}^{(0)} \\
\mathbf{Q}^{\star} & =\boldsymbol{\Phi}^{T} \mathbf{J} \mathbf{u}^{(N)}=\boldsymbol{\Phi}_{q}^{T} \mathbf{F}^{(N)}-\boldsymbol{\Phi}_{F}^{T} \mathbf{q}^{(N)}
\end{aligned}
$$

Therefore, by combining equations (3), (4), (5), (7), and (8), we obtain

$$
\begin{aligned}
\mathbf{q}^{(n)}= & -\boldsymbol{\Phi}_{q} \boldsymbol{\mu}^{n} \boldsymbol{\Phi}_{F}^{\star T} \mathbf{q}^{(0)}+\boldsymbol{\Phi}_{q}^{\star} \boldsymbol{\mu}^{N-n} \boldsymbol{\Phi}_{F}^{T} \mathbf{q}^{(N)} \\
& +\boldsymbol{\Phi}_{q} \sum_{k=0}^{n} \boldsymbol{\mu}^{n-k} \boldsymbol{\Phi}_{q}^{\star T}\left(\mathbf{F}_{E}^{(k)}+\tilde{\mathbf{F}}^{(k)}\right)+\boldsymbol{\Phi}_{q}^{\star} \sum_{k=n+1}^{N} \boldsymbol{\mu}^{k-n} \boldsymbol{\Phi}_{q}^{T}\left(\mathbf{F}_{E}^{(k)}+\tilde{\mathbf{F}}^{(k)}\right)
\end{aligned}
$$

where $\tilde{\mathbf{F}}^{(0)}=\mathbf{F}_{\partial L}$ and $\tilde{\mathbf{F}}^{(N)}=\mathbf{F}_{\partial R}$. We can rewrite the aforementioned equation in matrix form as follows

$$
\begin{aligned}
{\left[\begin{array}{ccccc}
\mathbf{I}+\boldsymbol{\Phi}_{q} \boldsymbol{\Phi}_{F}^{\star T} & 0 & 0 & 0 & -\boldsymbol{\Phi}_{q}^{\star} \boldsymbol{\mu}^{N} \boldsymbol{\Phi}_{F}^{T} \\
\vdots & \ddots & \vdots & \vdots & \vdots \\
\boldsymbol{\Phi}_{q} \boldsymbol{\mu}^{n} \boldsymbol{\Phi}_{F}^{\star T} & 0 & \mathbf{I} & 0 & -\boldsymbol{\Phi}_{q}^{\star} \boldsymbol{\mu}^{N-n} \boldsymbol{\Phi}_{F}^{T} \\
\vdots & \vdots & \vdots & \ddots & \vdots \\
\boldsymbol{\Phi}_{q} \boldsymbol{\mu}^{N} \boldsymbol{\Phi}_{F}^{\star T} & 0 & 0 & 0 & \mathbf{I}-\boldsymbol{\Phi}_{q}^{\star} \boldsymbol{\Phi}_{F}^{T}
\end{array}\right]\left[\begin{array}{c}
\mathbf{q}^{(0)} \\
\vdots \\
\mathbf{q}^{(n)} \\
\vdots \\
\mathbf{q}^{(N)}
\end{array}\right] } \\
=\left[\begin{array}{ccccc}
\boldsymbol{\Phi}_{q} \boldsymbol{\Phi}_{q}^{\star T} & \cdots & \boldsymbol{\Phi}_{q}^{\star} \boldsymbol{\mu}^{n} \boldsymbol{\Phi}_{q}^{T} & \cdots & \boldsymbol{\Phi}_{q}^{\star} \boldsymbol{\mu}^{N} \boldsymbol{\Phi}_{q}^{T} \\
\vdots & \ddots & \vdots & \vdots & \vdots \\
\boldsymbol{\Phi}_{q} \boldsymbol{\mu}^{n} \boldsymbol{\Phi}_{q}^{\star T} & \cdots & \boldsymbol{\Phi}_{q} \boldsymbol{\Phi}_{q}^{\star T} & \cdots & \boldsymbol{\Phi}_{q}^{\star} \boldsymbol{\mu}^{N-n} \boldsymbol{\Phi}_{q}^{T} \\
\vdots & \vdots & \vdots & \ddots & \\
\boldsymbol{\Phi}_{q} \boldsymbol{\mu}^{N} \boldsymbol{\Phi}_{q}^{\star T} & \cdots & \boldsymbol{\Phi}_{q} \boldsymbol{\mu}^{N-n} \boldsymbol{\Phi}_{q}^{\star T} & \cdots & \boldsymbol{\Phi}_{q} \boldsymbol{\Phi}_{q}^{\star T}
\end{array}\right]\left[\begin{array}{c}
\mathbf{F}_{E}^{(0)}+\mathbf{F}_{\partial L} \\
\vdots \\
\mathbf{F}_{E}^{(n)}+\tilde{\mathbf{F}}_{\partial n} \\
\vdots \\
\mathbf{F}_{E}^{(N)}+\mathbf{F}_{\partial R}
\end{array}\right]
\end{aligned}
$$

Equation (10) is a relation between the DOF and nodal loads of the whole domain. Moreover, the matrix on the right side of equation (10) is a block Toeplitz matrix [13, 14].

\subsection{Assembly of boundary nodes}

We are interested in the boundary nodes of the domain. The left and right boundaries are $\mathbf{q}^{(0)}$ and $\mathbf{q}^{(N)}$. Otherwise, the up and down boundaries at the column $n$ (noted by $\partial n$ ) are the first and the last nodes of the column and we can define a matrix $\mathbf{L}$ so that:

$$
\mathbf{q}_{\partial n}=\mathbf{L} \mathbf{q}^{(n)}, \quad \mathbf{F}_{\partial n}=\mathbf{L} \tilde{\mathbf{F}}^{(n)} \quad 0<n<N
$$

If we note

$$
\tilde{\boldsymbol{\Phi}}_{q}=\mathbf{L} \boldsymbol{\Phi}_{q}, \quad \tilde{\boldsymbol{\Phi}}_{q}^{\star}=\mathbf{L} \boldsymbol{\Phi}_{q}^{\star}
$$


Equation (10) become

$$
\begin{aligned}
& {\left[\begin{array}{ccccc}
\mathbf{I}+\boldsymbol{\Phi}_{q} \boldsymbol{\Phi}_{F}^{\star T} & 0 & 0 & 0 & -\boldsymbol{\Phi}_{q}^{\star} \boldsymbol{\mu}^{N} \boldsymbol{\Phi}_{F}^{T} \\
\vdots & \ddots & \vdots & \vdots & \vdots \\
\tilde{\boldsymbol{\Phi}}_{q} \boldsymbol{\mu}^{n} \boldsymbol{\Phi}_{F}^{\star T} & 0 & \tilde{\mathbf{I}} & 0 & -\tilde{\boldsymbol{\Phi}}_{q}^{\star} \boldsymbol{\mu}^{N-n} \boldsymbol{\Phi}_{F}^{T} \\
\vdots & \vdots & \vdots & \ddots & \vdots \\
\boldsymbol{\Phi}_{q} \boldsymbol{\mu}^{N} \boldsymbol{\Phi}_{F}^{\star T} & 0 & 0 & 0 & \mathbf{I}-\boldsymbol{\Phi}_{q}^{\star} \boldsymbol{\Phi}_{F}^{T}
\end{array}\right]\left[\begin{array}{c}
\mathbf{q}_{\partial L} \\
\vdots \\
\mathbf{q}_{\partial n} \\
\vdots \\
\mathbf{q}_{\partial R}
\end{array}\right]} \\
& =\left[\begin{array}{ccccc}
\boldsymbol{\Phi}_{q} \boldsymbol{\Phi}_{q}^{\star T} & \ldots & \boldsymbol{\Phi}_{q}^{\star} \boldsymbol{\mu}^{n} \tilde{\boldsymbol{\Phi}}_{q}^{T} & \ldots & \boldsymbol{\Phi}_{q}^{\star} \boldsymbol{\mu}^{N} \boldsymbol{\Phi}_{q}^{T} \\
\vdots & \ddots & \vdots & \vdots & \vdots \\
\tilde{\boldsymbol{\Phi}}_{q} \boldsymbol{\mu}^{n} \boldsymbol{\Phi}_{q}^{\star T} & \ldots & \tilde{\boldsymbol{\Phi}}_{q} \tilde{\boldsymbol{\Phi}}_{q}^{\star T} & \ldots & \tilde{\boldsymbol{\Phi}}_{q}^{\star} \boldsymbol{\mu}^{N-n} \boldsymbol{\Phi}_{q}^{T} \\
\vdots & \vdots & \vdots & \ddots & \\
\boldsymbol{\Phi}_{q} \boldsymbol{\mu}^{N} \boldsymbol{\Phi}_{q}^{\star T} & \cdots & \boldsymbol{\Phi}_{q} \boldsymbol{\mu}^{N-n} \tilde{\boldsymbol{\Phi}}_{q}^{\star T} & \ldots & \boldsymbol{\Phi}_{q} \boldsymbol{\Phi}_{q}^{\star T}
\end{array}\right]\left[\begin{array}{c}
\mathbf{F}_{\partial L} \\
\vdots \\
\mathbf{F}_{\partial n} \\
\vdots \\
\mathbf{F}_{\partial R}
\end{array}\right] \\
& +\left[\begin{array}{ccccc}
\boldsymbol{\Phi}_{q} \boldsymbol{\Phi}_{q}^{\star T} & \cdots & \boldsymbol{\Phi}_{q}^{\star} \boldsymbol{\mu}^{n} \boldsymbol{\Phi}_{q}^{T} & \cdots & \boldsymbol{\Phi}_{q}^{\star} \boldsymbol{\mu}^{N} \boldsymbol{\Phi}_{q}^{T} \\
\vdots & \ddots & \vdots & \vdots & \vdots \\
\tilde{\boldsymbol{\Phi}}_{q} \boldsymbol{\mu}^{n} \boldsymbol{\Phi}_{q}^{\star T} & \ldots & \tilde{\boldsymbol{\Phi}}_{q} \boldsymbol{\Phi}_{q}^{\star T} & \cdots & \tilde{\boldsymbol{\Phi}}_{q}^{\star} \boldsymbol{\mu}^{N-n} \boldsymbol{\Phi}_{q}^{T} \\
\vdots & \vdots & \vdots & \ddots & \\
\boldsymbol{\Phi}_{q} \boldsymbol{\mu}^{N} \boldsymbol{\Phi}_{q}^{\star T} & \cdots & \boldsymbol{\Phi}_{q} \boldsymbol{\mu}^{N-n} \boldsymbol{\Phi}_{q}^{\star T} & \cdots & \boldsymbol{\Phi}_{q} \boldsymbol{\Phi}_{q}^{\star T}
\end{array}\right]\left[\begin{array}{c}
\mathbf{F}_{E}^{(0)} \\
\vdots \\
\mathbf{F}_{E}^{(n)} \\
\vdots \\
\mathbf{F}_{E}^{(N)}
\end{array}\right]
\end{aligned}
$$

where $\tilde{\mathbf{I}}=\mathbf{L I L}^{T}$ which is also an identity matrix. We can rewrite the aforementioned equation as follows

$$
\mathbb{A} \mathbf{q}_{\partial}=\mathbb{B} \mathbf{F}_{\partial}+\mathbb{C F}_{E}
$$

where $\mathbb{A}, \mathbb{B}, \mathbb{C}$ are defined corresponding to the matrices in equation (13).

\subsection{Assembly of the whole structure}

For the domain $\mathcal{R}$, we have

$$
\left[\begin{array}{lll}
\mathbf{D}_{\partial \partial}^{\star} & \mathbf{D}_{\partial I}^{\star} & \mathbf{D}_{\partial B}^{\star} \\
\mathbf{D}_{I \partial}^{\star} & \mathbf{D}_{I I}^{\star} & \mathbf{D}_{I B}^{\star} \\
\mathbf{D}_{B \partial}^{\star} & \mathbf{D}_{B I}^{\star} & \mathbf{D}_{B B}^{\star}
\end{array}\right]\left[\begin{array}{l}
\mathbf{q}_{\partial} \\
\mathbf{q}_{I} \\
\mathbf{q}_{0}
\end{array}\right]=\left[\begin{array}{c}
\mathbf{F}_{\partial} \\
\mathbf{F}_{0}^{\star} \\
\mathbf{F}_{B}^{\star}
\end{array}\right]
$$

where $\mathbf{q}_{0}, \mathbf{F}_{0}^{\star}$ are given by the boundary condition and the loads of the domain $\mathcal{R}$. From the aformentioned equation, we obtain

$$
\left[\begin{array}{ll}
\mathbf{D}_{\partial \partial}^{\star} & \mathbf{D}_{\partial I}^{\star} \\
\mathbf{D}_{I \partial}^{\star} & \mathbf{D}_{I I}^{\star}
\end{array}\right]\left[\begin{array}{l}
\mathbf{q}_{\partial} \\
\mathbf{q}_{I}
\end{array}\right]=\left[\begin{array}{l}
\mathbf{F}_{\partial} \\
\mathbf{F}_{0}^{\star}
\end{array}\right]-\left[\begin{array}{l}
\mathbf{D}_{\partial B}^{\star} \mathbf{q}_{0} \\
\mathbf{D}_{I B}^{\star} \mathbf{q}_{0}
\end{array}\right]
$$

Equations (14) and (16) decribe the relations between $\mathbf{q}_{\partial}$ and $\mathbf{F}_{\partial}$ with the external loads and the boundary conditions of the whole structure. We can have different methods to combine these two equations which cost different calculation times. It is possible to reduce the cost by using the properties of the Toeplizt block matrix $\mathbb{B}, \mathbb{C}[14]$. However, this article is limited to a simple method by substituting equation (14) into equation (16) and we obtain

$$
\left[\begin{array}{cc}
\mathbf{D}_{\partial \partial}^{\star}-\mathbb{B}^{-1} \mathbb{A} & \mathbf{D}_{\partial I}^{\star} \\
\mathbf{D}_{I \partial}^{\star} & \mathbf{D}_{I I}^{\star}
\end{array}\right]\left[\begin{array}{l}
\mathbf{q}_{\partial} \\
\mathbf{q}_{I}
\end{array}\right]=\left[\begin{array}{c}
-\mathbb{B}^{-1} \mathbb{C} \mathbf{F}_{E} \\
\mathbf{F}_{0}^{\star}
\end{array}\right]-\left[\begin{array}{c}
\mathbf{D}_{\partial B}^{\star} \mathbf{q}_{0} \\
\mathbf{D}_{I B}^{\star} \mathbf{q}_{0}
\end{array}\right]
$$


Equation (17) permits to calculate the response of the domain $\mathcal{R}$. This technique permits to get the final expression of the dynamic stiffness matrix (DSM) of the whole structure by modifying only the diagonal block of DSM of $\mathcal{R}$ corresponding to the rectangular domain boundary.

When the structure has severalrectangular domains, we can use the same technique for each diagonal block to get the final DSM. For structures with connected rectangular domains, we need to combine these domains as superelements before replacing into the DSM.

\section{EXAMPLE}

Let's consider a $2 \mathrm{D}$ elipse of axes $6 \mathrm{mx} 3 \mathrm{~m}$ which contain a rectangular of size $4 \mathrm{~m} \times 2 \mathrm{~m}$ as shown in Figure 4. The mesh is created by using 1586 elements $\mathrm{S} 4$ of thickness $0.1 \mathrm{~m}$. The stiffness and mass matrices are generated by Abaqus and other calculations have been performed with Matlab. Figure 5 shows the results obtained by the finite element method (for the whole

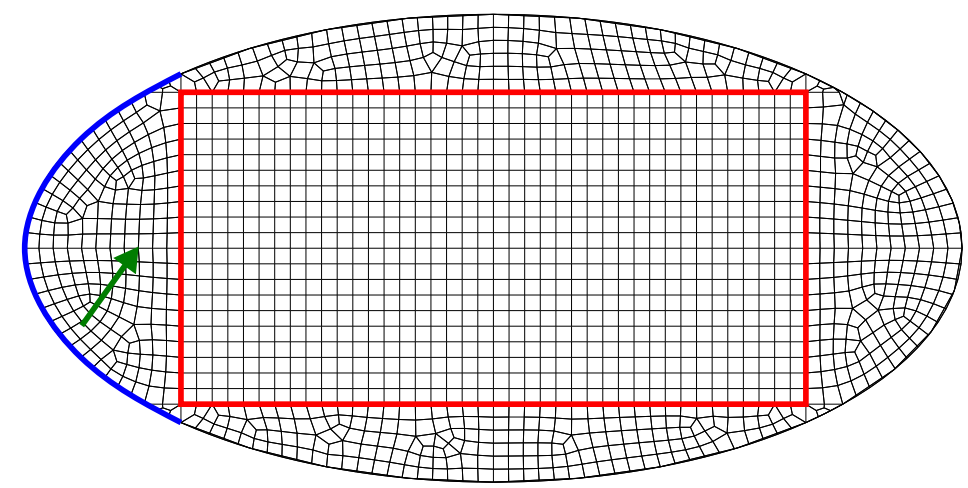

Figure 4: Example of 2D structure with a rectanglar domain (red), a fixed boundary (blue) and a load (green)

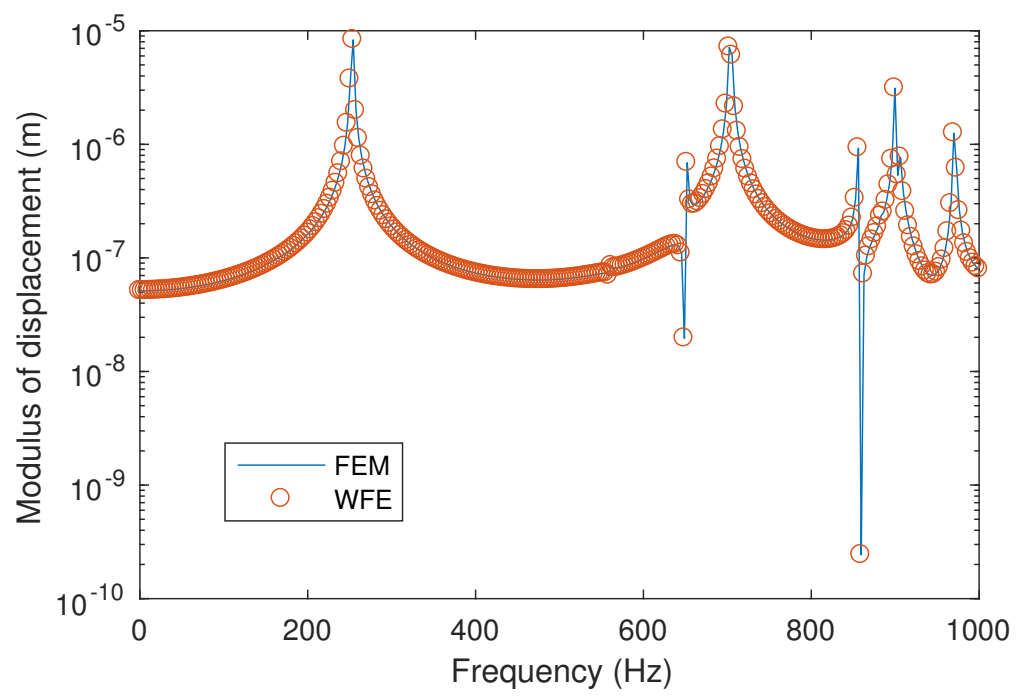

Figure 5: Response of one node calculated by the finite element method and the new method

structure) and the new method. The two results agrees well. Althought the new method can 
reduce the number of DOF (from 3322 to 1840), the WFE cannot reduce the calculation time in comparing with FEM (5.4s vs 20.9s). One reason is that the calculation of Toeplitz matrices (which are full matrices) have not been optimized.

\section{CONCLUSIONS}

This article introduces a new technique for coupling the finite element method and the wave finite element method by using superelements. For a 2D structure of arbtrary shapes, we define superelements as rectangular domains. Each superelement is a periodic structure where we can apply WFE. We obtain then a relation between the DOF at the superelement boundary and the external nodal loads via Toeplitz matrices. The technique permits to reduce the number of DOF but it needs to optimize the calculation of Toeplitz matrices in order to reduce the calculation time.

\section{REFERENCES}

[1] D. Mead, A general theory of harmonic wave propagation in linear periodic systems with multiple coupling, Journal of Sound and Vibration , 27, 235 - 260, 1973.

[2] D. Duhamel, B. R. Mace, M. J. Brennan, Finite element analysis of the vibrations of waveguides and periodic structures, Journal of Sound and Vibration, 294, 205-220, 2006.

[3] Y. Waki, B. Mace, M. Brennan, Free and forced vibrations of a tyre using a wave finite element approach, Journal of Sound and Vibration, 323, 737 - 756, 2009.

[4] W. Zhou, M. Ichchou, Wave propagation in mechanical waveguide with curved members using wave finite element solution, Computer Methods in Applied Mechanics and Engineering, 199, 2099 - 2109, 2010.

[5] M. Ichchou, J.-M. Mencik, W. Zhou, Wave finite elements for low and mid-frequency description of coupled structures with damage, Computer Methods in Applied Mechanics and Engineering, 198, 1311 - 1326, 2009.

[6] B. Lossouarn, M. Aucejo, J.-F. Deu, Electromechanical wave finite element method for interconnected piezoelectric waveguides, Computers \& Structures, 199, 46 - 56, 2018.

[7] A. Kessentini, M. Taktak, M. B. Souf, O. Bareille, M. Ichchou, M. Haddar, Computation of the scattering matrix of guided acoustical propagation by the wave finite element approach, Applied Acoustics, 108, 92 - 100, 2016.

[8] J.-M. Mencik, A wave finite element approach for the analysis of periodic structures with cyclic symmetry in dynamic substructuring, Journal of Sound and Vibration, 431, 441 457, 2018.

[9] T. Gras, M.-A. Hamdi, M. B. Tahar, O. Tanneau, L. Beaubatie, On a coupling between the finite element (fe) and the wave finite element (wfe) method to study the effect of a local heterogeneity within a railway track, Journal of Sound and Vibration, 429, 45 - 62, 2018.

[10] J.-M. Mencik, D. Duhamel, A wave finite element-based approach for the modeling of periodic structures with local perturbations, Finite Elements in Analysis and Design, 121, 40-51, 2016. 
[11] J. M. Mencik, D. Duhamel, A wave-based model reduction technique for the description of the dynamic behavior of periodic structures involving arbitrary-shaped substructures and large-sized finite element models, Finite Elements in Analysis and Design, 101, 1-14, 2015.

[12] T. Hoang, D. Duhamel, G. Foret, Wave finite element method for vibration of periodic structures subjected to external loads, 6th European Conference on Computational Mechanics (ECCM 6), Glasgow, UK, June 2018.

[13] A. Bottcher, S.M. Grudsky, Toeplitz Matrices - Asymptotic Linear Algebra and Functional Analysis, Hindustan Book Agency, 2000.

[14] W. F. Trench, Inversion of Toeplitz band matrices, Mathematics of Computation, 28, 10891095, 1974. 